\title{
MINIMAL CONVERGENCE SPACES
}

\author{
BY \\ D. C. KENT AND G. D. RICHARDSON
}

\begin{abstract}
We are primarily concerned with minimal $\mathbf{P}$ convergence spaces, where $P$ is one of the following convergence space properties: Hausdorff, $T_{2}, \lambda$-regular, $\lambda$-Urysohn, and first countable, $\lambda$ an infinite cardinal number. Our conclusions usually resemble the corresponding topological results, but with some deviations; for instance, a minimal Hausdorff convergence space is always compact, whereas a countable minimal regular convergence space need not be compact.
\end{abstract}

Introduction. Minimal topological spaces have been extensively studied by various authors (see references). We now extend this study to convergence spaces. The reader is asked to refer to [9] for basic definitions and terminology concerning convergence spaces. However, a brief summary will be given here.

A convergence structure $q$ on a set $S$ is defined to be a function from the set $F(S)$ of all filters on $S$ into the set of all subsets of $S$, satisfying the following conditions:

(1) $x \in q(\dot{x})$, all $x \in S$, where $\dot{x}$ is the principal ultrafilter containing $\{x\}$;

(2) if $\mathscr{F}$ and $\mathscr{G}$ are in $F(S)$ and $\mathscr{F} \geqq \mathscr{G}$, then $q(\mathscr{G}) \subset q(\mathscr{F})$;

(3) if $x \in q(\mathscr{F})$, then $x \in q(\mathscr{F} \cap \dot{x})$.

The pair $(S, q)$ is called a convergence space. If $x \in q(\mathscr{F})$, then we say that $\mathscr{F}$ $q$-converges to $x$. The filter $\mathscr{V}_{q}(x)$ obtained by intersecting all filters which $q$-converge to $x$ is called the q-neighborhood filter at $x$. If $\mathscr{V}_{q}(x) q$-converges to $x$ for each $x$ in $S$, then $q$ is called a pretopology, and $(S, q)$ a pretopological space. A convergence structure $q$ is called a pseudo-topology if $\mathscr{F} q$-converges to $x$ whenever each ultrafilter finer than $\mathscr{F} q$-converges to $x$. The pair $(S, q)$ is called a pseudo-topological space.

Let $C(S)$ be the set of all convergence structures on $S$. The set $C(S)$ is partially ordered in the following way: $p \leqq q$ provided $q(\mathscr{F}) \subset p(\mathscr{F})$ for each $\mathscr{F}$ in $F(S)$. If $A$ is a subset of $(S, q)$, then $x$ is in $\Gamma_{q}(A)$, the $q$-closure of $A$, if there exists an ultrafilter containing $A$ which $q$-converges to $x$.

A convergence space is said to be minimal relative to property $\mathbf{P}$ if the space has property $\mathbf{P}$ and every strictly coarser convergence structure on the same set fails to have this property. A space is said to be P-closed if it has property $\mathbf{P}$ and is closed in every extension space that has property $\mathrm{P}$. We have considered the

Presented to the Society, August 28, 1970; received by the editors May 4, 1970 and, in revised form, November 18, 1970.

AMS 1969 subject classifications. Primary 5422; Secondary 5410.

Key words and phrases. Convergence spaces, minimal $\mathrm{P}$ convergence spaces, P-closed convergence spaces, $\lambda$-regular convergence spaces, $\lambda$-Urysohn convergence spaces. 
following choices for P: Hausdorff, regular, Urysohn, and first countable. In all cases simple characterizations are obtained for minimal $\mathbf{P}$ and $\mathrm{P}$-closed convergence spaces; in most cases the characterizations are primitive in the sense that property $\mathbf{P}$ is not assumed as a precondition. Our conclusions usually resemble the corresponding topological results, but with some interesting deviations; for instance, a minimal Hausdorff convergence space is always compact (contrary to the topological case), whereas a countable minimal regular convergence space need not be compact (also contrary to the topological case).

For convergence spaces it is necessary to distinguish between Hausdorff (each filter converges to at most one point) and $\mathrm{T}_{2}$ (distinct points have disjoint neighborhoods), and we consider both properties but emphasize the former. Also, because the closure operator is not idempotent for most convergence spaces, the usual notion of regularity (see [3], [4], and [5]) seems rather inadequate; for this reason we are introducing a more general notion called $\lambda$-regularity ( $\lambda$ an infinite cardinal number) which reduces to ordinary regularity when $\lambda=\aleph_{0}$.

1. Minimal Hausdorff $\left(\mathrm{T}_{2}\right)$ convergence spaces. The term space, unless otherwise indicated, will mean convergence space. If $\mathscr{F}$ and $\mathscr{G}$ are filters on a set $S$, then $\mathscr{F} \vee \mathscr{G}$ denotes the coarsest filter finer than $\mathscr{F}$ and $\mathscr{G}$, if such a filter exists; otherwise we write $\mathscr{F} \vee \mathscr{G}=\varnothing$, and the filters are said to be disjoint. Thus a space $(S, q)$ is $\mathrm{T}_{2}$ if and only if $\mathscr{V}_{q}(x) \vee \mathscr{V}_{q}(y)=\varnothing$ for every pair $x, y$ of distinct points in $S$. A point $x$ is an adherent point of a filter if some finer filter converges to $x$; we denote by $\alpha_{q}(\mathscr{F})$ the set of all adherent points of $\mathscr{F}$ relative to the convergence structure $q$.

THEOREM 1.1. The following statements about a space are equivalent:

(i) $(S, q)$ is minimal Hausdorff.

(ii) $\{x\}=\alpha_{q}(\mathscr{F})$ iff $\mathscr{F} q$-converges to $x$.

(iii) $(S, q)$ is a compact pseudo-topological Hausdorff space.

Proof. (i) implies (ii). If $\{x\}=\alpha_{q}(\mathscr{F})$ and $\mathscr{F}$ fails to $q$-converge to $x$, then define the convergence structure $p$ on $S$ as follows: $\mathscr{H} p$-converges to $x$ iff $\mathscr{H} \geqq \mathscr{G} \cap \mathscr{F}$ where $\mathscr{G} q$-converges to $x$; $\mathscr{H} p$-converges to $y, y \neq x$, iff $\mathscr{H} q$-converges to $y$. But $p$ is Hausdorff and strictly coarser than $q$, a contradiction. Hence $\mathscr{F} q$-converges to $x$.

(ii) implies (iii). If $\mathscr{F}$ is an ultrafilter such that $\alpha_{q}(\mathscr{F})=\varnothing$, then $\alpha_{q}(\mathscr{F} \cap \dot{x})=\{x\}$. Hence $\mathscr{F} q$-converges to $x$, a contradiction, and it follows that $(S, q)$ is compact. If each ultrafilter finer than $\mathscr{G} q$-converges to $x$, then $\{x\}=\alpha_{q}(\mathscr{G})$, and by (ii), $\mathscr{G}$ $q$-converges to $x$. Thus $(S, q)$ is a pseudo-topology.

(iii) implies (i). If $p$ is a Hausdorff convergence structure coarser than $q$ and $\mathscr{F} p$-converges to $x$, then $\{x\}=\alpha_{q}(\mathscr{F})$. Let $\mathscr{G}$ be an ultrafilter finer than $\mathscr{F}$; then $\mathscr{G} q$-converges to $x$. Thus $\mathscr{F} q$-converges to $x$, and $p=q$.

THEOREM 1.2. The following are equivalent:

(i) $(S, q)$ is minimal $\mathrm{T}_{2}$.

(ii) $(S, q)$ is $\mathrm{T}_{2}$ and $\{x\}=\alpha_{\pi(q)}(\mathscr{F})$ implies that $\mathscr{F} q$-converges to $x$. 
(iii) $(S, q)$ is a compact $\mathrm{T}_{2}$ pretopological space.

(iv) (a) For each ultrafilter $\mathscr{F}$ there exists at most one $x$ in $S$ with $\dot{x} \geqq \Gamma(\mathscr{F})$.

(b) $\{x\}=\alpha_{q}(\mathscr{F})$ implies that $\mathscr{F} q$-converges to $x$.

Note. $\Gamma(\mathscr{F})$ denotes the closure of $\mathscr{F}$, i.e. the filter generated by closures of members of $\mathscr{F}$.

Proof. The arguments for (i) implies (ii) and (ii) implies (iii) are similar to those of the preceding theorem.

(iii) implies (iv). Let $\mathscr{F}$ be an ultrafilter and suppose $\dot{x} \geqq \Gamma(\mathscr{F})$ and $\dot{y} \geqq \Gamma(\mathscr{F})$. Then $\mathscr{F} \vee \mathscr{V}_{q}(x) \neq \varnothing$. Hence $\mathscr{F} \geqq \mathscr{V}_{q}(x)$; similarly, $\mathscr{F} \geqq \mathscr{V}_{q}(y)$, and we have $\mathscr{V}_{q}(x) \vee \mathscr{V}_{q}(y) \neq \varnothing$, a contradiction. Part (b) follows from Theorem 1.1.

(iv) implies (i). If $\mathscr{V}_{q}(x) \vee \mathscr{V}_{q}(y) \neq \varnothing$, then there is an ultrafilter $\mathscr{G} \geqq \mathscr{V}_{q}(x) \vee \mathscr{V}_{q}(y)$. Hence $\dot{x} \geqq \Gamma(\mathscr{G})$ and $\dot{y} \geqq \Gamma(\mathscr{G})$, a contradiction. Thus $q$ is $\mathrm{T}_{2}$. If $p \leqq q$ where $p$ is $\mathrm{T}_{2}$, then let $\mathscr{F} p$-converge to $x$. Then $\{x\}=\alpha_{q}(\mathscr{F} \cap \dot{x})$ and consequently $\mathscr{F} q$-converges to $x$, and $p=q$.

Corollary 1.3. A Hausdorff topological space is minimal Hausdorff $\left(\mathrm{T}_{2}\right)$ iff it is compact.

Here, as always, we use "minimal" in the convergence space sense unless otherwise indicated.

Proposition 1.4. Let $(S, q)$ be a minimal Hausdorff $\left(\mathrm{T}_{2}\right)$ space. Then a subset $A$ of $S$ is closed iff the subspace $\left(A, q_{A}\right)$ is minimal Hausdorff $\left(\mathrm{T}_{2}\right)$. Moreover, only the Hausdorff $\left(\mathrm{T}_{2}\right)$ property is needed for the sufficiency.

Corollary 1.5. A minimal Hausdorff $\left(\mathrm{T}_{2}\right)$ space is Hausdorff $\left(\mathrm{T}_{2}\right)$-closed.

THEOREM 1.6. A Hausdorff $\left(\mathrm{T}_{2}\right)$ space is Hausdorff $\left(\mathrm{T}_{2}\right)$-closed iff $(S, q)((S, \pi(q)))$ is compact.

Proof. If $(S, q)$ is Hausdorff-closed and not compact, then there exists a filter $\mathscr{F}$ with $\alpha_{q}(\mathscr{F})=\varnothing$. Let $a$ be an element not in $S$ and $T=S \cup\{a\}$. Define a convergence structure $p$ on $T$ as follows: $\mathscr{G} p$-converges to $a$ iff $\mathscr{G} \geqq \mathscr{F} \cap \dot{a} ; \mathscr{G} p$-converges to $x$ in $S$ iff $\mathscr{G} q$-converges to $x$. But $p$ is Hausdorff and $\Gamma(S)=T$, a contradiction. Thus $(S, q)$ is compact.

The converse is clear. The $T_{2}$ case is handled similarly.

We now given an example of a minimal Hausdorff space which is not $\mathrm{T}_{2}$-closed.

EXAMPLE 1.7. Let $S$ be any infinite set, $\mathscr{F}$ some free ultrafilter on $S$. We define a Hausdorff pseudo-topology $q$ by designating convergence on ultrafilters as follows: for each $x, \dot{x} q$-converges only to $x$; if $\mathscr{G}$ is a free ultrafilter distinct from $\mathscr{F}$, then $\mathscr{G} q$-converges to a fixed element $x_{0} ; \mathscr{F} q$-converges to another fixed element $y_{0}$. Then $q$ is a compact Hausdorff pseudo-topology, but $\mathscr{F} \geqq \mathscr{V}_{q}\left(x_{0}\right)$ $\vee \mathscr{V}_{q}\left(y_{0}\right)$, and so $q$ is not $\mathrm{T}_{2}$.

THEOREM 1.8. Let $(S, q)=\prod\left\{\left(S_{i}, q_{i}\right)\right\}$, where $i$ ranges through some index set. Then the product space $(S, q)$ is minimal Hausdorff (minimal $\mathrm{T}_{2}$ ) iff each $\left(S_{i}, q_{i}\right)$ is minimal Hausdorff (minimal $\mathrm{T}_{2}$ ). 
Proof. One can show by simple arguments that $(S, q)$ is Hausdorff (compact, pseudo-topological, pretopological) iff each $\left(S_{i}, q_{i}\right)$ is Hausdorff (compact, pseudotopological, pretopological). The desired conclusions thus follow from Theorems 1.1 and 1.2.

Proposition 1.9. Let $f:(S, q) \rightarrow(T, p)$ be a continuous bijection with $(T, p)$ Hausdorff and $(S, q)$ minimal Hausdorff. Then $f$ is an isomorphism. The proposition is no longer true if minimal Hausdorff is replaced by compact Hausdorff.

Proof. Let $\mathscr{F} p$-converge to $y$ in $T$. Then we easily see that there is $x$ in $S$ such that $\{x\}=\alpha_{q}\left(f^{-1}(\mathscr{F})\right)$; hence $f^{-1}(\mathscr{F}) q$-converges to $x$. Thus $f$ is an isomorphism. The second assertion follows from the next example.

EXAMPLE 1.10. Let $S$ be the closed unit interval on the real line and let $q$ be defined as follows: $\mathscr{F} q$-converges to $x$ iff $\mathscr{F} \geqq \mathscr{G} \cap \dot{x}$, where $\mathscr{G}$ is an ultrafilter which converges to $x$ in the usual sense. We see that $q$ is compact Hausdorff and strictly finer than the usual topology $p$ on $S$. The identity mapping is a continuous bijection from $(S, q)$ onto $(S, p)$, but it is not an isomorphism.

The next result is a consequence of Theorem 1.1 and Proposition 1.9.

Corollary 1.11 (Theorem 7, P. 284 of [5]). Let $f:(S, q) \rightarrow(T, p)$ be a continuous bijection with $(T, p)$ Hausdorff and $(S, q)$ a compact pretopology. Then $f$ is an isomorphism.

Proposition 1.12. Let $(S, q)$ be any Hausdorff space. Then there is a minimal Hausdorff convergence structure on $S$ coarser than $q$.

Proof. Let $x$ be a fixed element of $S$. Define $p$ as follows: $\mathscr{H} p$-converges to $x$ iff either $\mathscr{H} q$-converges to $x$ or $\mathscr{H} \geqq \mathscr{F} \cap \dot{x}$, where $\mathscr{F}$ is a $q$-nonconvergent ultrafilter; $\mathscr{H} p$-converges to $y, y \neq x$, iff $\mathscr{H} q$-converges to $y$. Clearly $(S, p)$ is Hausdorff. Next, define $r$ as follows: $\mathscr{G} r$-converges to $z$ in $S$ iff, for each ultrafilter $\mathscr{H} \geqq \mathscr{G}, \mathscr{H}$ $p$-converges to $z$. Note that $r$ is a compact Hausdorff pseudo-topology and that $r \leqq q$.

That the preceding proposition is not valid for topological spaces is shown in Theorem 21 of [7]. More surprisingly, the proposition is false even for convergence spaces if "Hausdorff" is replaced by " $\mathrm{T}_{2}$ ". An argument similar to that used by Herrlich in Theorem 21 of [7] can be used to produce a counterexample.

2. Minimal regular spaces. Let $(S, q)$ be a Hausdorff convergence space. In all of the definitions in this and succeeding sections it will be assumed that the underlying space is Hausdorff; the spaces are not assumed to be $T_{2}$ unless so indicated.

In $\S \S 2$ and 3 we will use $\lambda$ to represent an arbitrary cardinal number, and $\lambda^{*}$ to be the smallest ordinal number of cardinality $\lambda$. One departure from this notation is the use of $\omega$ as the smallest ordinal of cardinality $\boldsymbol{N}_{0}$.

If $\mathscr{F}$ is any filter on $S$ and $\sigma$ an ordinal number greater than 1 , we define $\Gamma^{\sigma}(\mathscr{F})$ recursively to be the closure of $\Gamma^{\sigma-1}(\mathscr{F})$, if $\sigma-1$ exists, or otherwise $\bigcap\left\{\Gamma^{\rho}(\mathscr{F}): \rho<\sigma\right\}$. If there is a possibility of confusion, we write $\Gamma_{q}^{\sigma}(\mathscr{F})$ to emphasize that the $\sigma$-closure is taken relative to the convergence structure $q$. 
Definition 2.1. A space $(S, q)$ is $\lambda$-regular if, for all $\sigma<\lambda^{*}, \Gamma^{\sigma}(\mathscr{F})$ converges to $x$ whenever $\mathscr{F}$ converges to $x$. A $\mathrm{T}_{2} \lambda$-regular space is said to be $\lambda$-T $\mathrm{T}_{3}$. An $\boldsymbol{\kappa}_{0}$ regular $\left(\boldsymbol{N}_{0}-\mathrm{T}_{3}\right)$ space is simply referred to as regular $\left(\mathrm{T}_{3}\right)$.

This definition of regularity agrees with the one which was introduced by Fischer [5] in 1959. An apparently more complicated notion of regularity which was introduced by Cook and Fischer [4] was later shown by Biesterfeldt [3] to be equivalent to Fischer's original definition. Its subsequent adoption by various authors justifies our calling it the standard definition of regularity for convergence spaces, although it is by no means the only possible one; for a discussion of some alternatives, see [6].

For each filter $\mathscr{F}$ on $S$ there is a smallest ordinal $\gamma$ such that $\Gamma^{\gamma+1}(\mathscr{F})=\Gamma^{\gamma}(\mathscr{F})$; we write $\Gamma^{\gamma}(\mathscr{F})=\hat{\mathscr{F}}$. A filter $\mathscr{F}$ is said to be closed if it coincides with $\hat{\mathscr{F}}$.

DEFINITION 2.2. A space is strongly regular if $\hat{\mathscr{F}}$ converges to $x$ whenever $\mathscr{F}$ converges to $x$. $\mathrm{A} \mathrm{T}_{2}$ strongly regular space is said to be strongly $\mathrm{T}_{3}$.

In other words, a space is strongly regular iff it is $\lambda$-regular for all $\lambda$. For topological spaces, the various forms of regularity and the $T_{3}$ property introduced in Definitions 2.1 and 2.2 are equivalent to each other and to the usual topological notion of regularity.

In the theorems that follow, we shall prove only those statements pertaining to regularity; the corresponding $\mathrm{T}_{3}$ statements, when known, are stated without proof.

Lemma 2.3. Let $(S, q)$ be $\lambda$-regular, and suppose that $\mathscr{F} q$-converges to $x$. Then $\{x\}=\alpha_{q}\left(\Gamma^{\lambda^{*}}(\mathscr{F})\right)$.

Proof. If $y \neq x$ and $\mathscr{G} q$-converges to $y$, then by the $\lambda$-regularity of $q, \Gamma(\mathscr{G})$ $q$-converges to $y$, and so there is $G$ in $\mathscr{G}$ such that $x \notin \Gamma(G)$. Thus for each $\sigma<\lambda^{*}$, there is $A_{\sigma} \in \Gamma^{\sigma}(\mathscr{F})$ such that $G \cap A_{\sigma}=\varnothing$, and it follows that $A=\bigcup A_{\sigma} \in \Gamma^{\lambda^{*}}(\mathscr{F})$ and $A \cap G=\varnothing$. Hence $\{x\}=\alpha_{q}\left(\Gamma^{\lambda^{*}}(\mathscr{F})\right)$.

THEOREM 2.4. The following statements about a convergence space $(S, q)$ are equivalent:

(i) $(S, q)$ is minimal $\lambda$-regular.

(ii) $\{x\}=\alpha_{q}\left(\Gamma^{\lambda^{*}}(\mathscr{F})\right)$ iff $\mathscr{F} q$-converges to $x$.

Proof. (i) implies (ii). Let $\{x\}=\alpha_{q}\left(\Gamma^{\lambda^{*}}(\mathscr{F})\right)$ and suppose that $\mathscr{F}$ does not $q$ converge to $x$. Let $p$ be defined as follows: $\mathscr{H} p$-converges to $x$ iff $\mathscr{H} \geqq \Gamma_{q}^{\sigma}(\mathscr{F}) \cap \mathscr{G}$, where $\mathscr{G} q$-converges to $x$ and $\sigma<\lambda^{*} ; \mathscr{H} p$-converges to $y, y \neq x$, iff $\mathscr{H} q$-converges to $y$. We will obtain a contradiction by showing that $p$ is $\lambda$-regular. Let $\mathscr{H} p$ converge to $x$. If $\rho<\lambda^{*}$, then $\Gamma_{p}^{o}(\mathscr{H}) \geqq \Gamma_{q}^{\sigma+\rho}(\mathscr{F}) \cap \Gamma_{q}^{o}(\mathscr{G})$ and thus $\Gamma_{p}^{o}(\mathscr{H}) p$-converges to $x$. Let $\mathscr{H} p$-converge to $y$. Then $\Gamma_{p}^{o}(\mathscr{H})=\Gamma_{q}^{o}(\mathscr{H})$ or $\Gamma_{p}^{o}(\mathscr{H})=\Gamma_{q}^{o}(\mathscr{H}) \cap \dot{x}$; since $\Gamma_{q}^{o}(\mathscr{H}) q$-converges to $y$ by the $\lambda$-regularity of $q$, it remains to show that $\dot{x} \geq \Gamma_{p}^{o}(\mathscr{H})$. Suppose the contrary, and let $\gamma$ be the least ordinal such that $\dot{x} \geqq \Gamma_{p}^{\gamma}(\mathscr{H})$. If $\gamma-1$ exists, then $\Gamma_{p}^{\gamma-1}(\mathscr{H})=\Gamma_{q}^{\gamma-1}(\mathscr{H})$, and this filter $q$-converges to $y$. Since $\dot{x} \geq \Gamma_{q}^{\gamma}(\mathscr{H})$, there is a set $A \in \Gamma_{p}^{\gamma-1}(\mathscr{H})$ such that each ultrafilter $\mathscr{G}$ which $q$-converges to $x$ fails 
to contain $A$. Also, $\{x\}=\alpha_{q}\left(\Gamma^{\lambda^{*}}(\mathscr{F})\right)$ implies that $\Gamma_{p}^{\gamma-1}(\mathscr{H})$ contains a set $B$ which is disjoint from some set in $\Gamma^{\lambda^{*}}(\mathscr{F})$. Thus no filter which $p$-converges to $x$ can contain $A \cap B$, and $x \notin \Gamma(A \cap B) \in \Gamma_{p}^{\gamma}(\mathscr{H})$, a contradiction. If $\gamma$ is a limit ordinal, then for each $\sigma<\gamma$ there is a set $A_{\sigma} \in \Gamma_{p}^{\sigma}(\mathscr{H})$ such that $x \notin A_{\sigma}$. Hence $x \notin \bigcup A_{\sigma}$ $\in \Gamma_{p}^{\gamma}(\mathscr{H})$, a contradiction. Consequently, $\mathscr{F} q$-converges to $x$.

The converse follows from Lemma 2.3.

(ii) implies (i). First we check that $(S, q)$ is $\lambda$-regular. Let $\mathscr{F} q$-converge to $x$ and $\sigma<\lambda^{*}$. Then $\{x\}=\alpha_{q}\left(\Gamma^{\lambda^{*}}(\mathscr{F})\right)=\alpha_{q}\left(\Gamma^{\sigma+\lambda^{*}}(\mathscr{F})\right)$, and hence $\Gamma^{\sigma}(\mathscr{F}) q$-converges to $x$. Next, let $p$ be $\lambda$-regular with $p \leqq q$, and let $\mathscr{F} p$-converge to $x$. By Lemma 2.3 , it follows that $\{x\}=\alpha_{p}\left(\Gamma_{p}^{\lambda^{*}}(\mathscr{F})\right)$. Thus $\{x\}=\alpha_{q}\left(\Gamma_{q}^{\lambda^{*}}(\mathscr{F}) \cap \dot{x}\right)=\alpha_{q}\left(\Gamma_{q}^{\lambda^{*}}(\mathscr{F} \cap \dot{x})\right)$ and we have that $\mathscr{F} q$-converges to $x$. Consequently, $p=q$.

THEOREM 2.4*. The following statements about a $\mathrm{T}_{2}$ convergence space $(S, q)$ are equivalent:

(i) $(S, q)$ is a minimal $\lambda-\mathrm{T}_{3}$.

(ii) $\{x\}=\alpha_{\pi(q)}\left(\Gamma^{\lambda^{*}}(\mathscr{F})\right)$ iff $\mathscr{F} q$-converges to $x$.

The first corollary is obtained by taking $\lambda=\boldsymbol{\aleph}_{0}$, the second by taking $\lambda$ to be any cardinal number greater than the cardinality of the least ordinal $\gamma$ such that $\Gamma^{\gamma}(\mathscr{F})=\Gamma^{\gamma+1}(\mathscr{F})$ for all filters $\mathscr{F}$ on $S$; for such a $\lambda$ we have $\Gamma^{\lambda}(\mathscr{F})=\hat{\mathscr{F}}$.

Corollary 2.5. The following statements about a $\left(\mathrm{T}_{2}\right)$ convergence space $(S, q)$ are equivalent:

(i) $(S, q)$ is minimal regular (minimal $\mathrm{T}_{3}$ ).

(ii) $\{x\}=\alpha_{q}\left(\Gamma^{\omega}(\mathscr{F})\right)\left(\{x\}=\alpha_{\pi(q)}\left(\Gamma^{\omega}(\mathscr{F})\right)\right)$ iff $\mathscr{F} q$-converges to $x$.

COROLLARY 2.6. The following statements about a $\left(\mathrm{T}_{2}\right)$ convergence space are equivalent:

(i) $(S, q)$ is minimal strongly regular (minimal strongly $\mathrm{T}_{3}$ ).

(ii) $\{x\}=\alpha_{q}(\hat{\mathscr{F}})\left(\{x\}=\alpha_{\pi(q)}(\hat{\mathscr{F}})\right)$ iff $\mathscr{F}$ q-converges to $x$.

We have from Theorems 2.4 and $2.4^{*}$ that if $(S, q)$ is $\lambda$-regular $\left(\lambda-\mathrm{T}_{3}\right)$, then it is minimal $\lambda$-regular (minimal $\lambda$-T $\left.\mathrm{T}_{3}\right)$ iff $\{x\}=\alpha_{q}\left(\Gamma^{\lambda^{*}}(\mathscr{F})\right)\left(\{x\}=\alpha_{\pi(q)}\left(\Gamma^{\lambda^{*}}(\mathscr{F})\right)\right)$ implies that $\mathscr{F} q$-converges to $x$.

THEOREM 2.7. A compact $\lambda$-regular $\left(\lambda-\mathrm{T}_{3}\right)$ pseudo-topological space is minimal $\lambda$-regular (minimal $\lambda-\mathrm{T}_{3}$ ).

Proof. If $\{x\}=\alpha_{q}\left(\Gamma^{\lambda^{*}}(\mathscr{F})\right)$ and if $\mathscr{G}$ is an ultrafilter finer than $\mathscr{F}$, then $\mathscr{G} q$ converges to $x$. Since $q$ is a pseudo-topology, $\mathscr{F} q$-converges to $x$. Hence the result follows from our preceding remarks.

An example in $\$ 3$ shows that there are noncompact pseudo-topological spaces of any infinite cardinality which are minimal $\lambda$-regular for all $\lambda$. The next theorem is also a consequence of the above remarks.

THEOREM 2.8. A minimal $\lambda$-regular (minimal $\lambda$-T $\mathrm{T}_{3}$ ) space is compact iff, for each ultrafilter $\mathscr{F}$, there is $x$ in $S$ such that $\{x\}=\alpha_{q}\left(\Gamma^{\lambda^{*}}(\mathscr{F})\right)\left(\{x\}=\alpha_{\pi(q)}\left(\Gamma^{\lambda^{*}}(\mathscr{F})\right)\right)$. 
A pretopological space is minimal $\lambda$-regular iff it is minimal $\lambda-T_{3}$.

Proposition 2.9. Let $(S, q)$ be minimal $\lambda$-regular. A subset $A$ of $S$ is closed iff the subspace $\left(A, q_{A}\right)$ is minimal $\lambda$-regular. Moreover, only $\lambda$-regularity is needed for sufficiency. The proposition remains valid if " $\lambda$-regular" is replaced by " $\lambda-\mathrm{T}_{3}$ ".

THEOREM 2.10. A $\lambda$-regular $\left(\lambda-\mathrm{T}_{3}\right)$ space $(S, q)$ is $\lambda$-regular-closed $\left(\lambda-\mathrm{T}_{3}\right.$-closed $)$ iff $\alpha_{q}\left(\Gamma^{\lambda^{*}}(\mathscr{F})\right) \neq \varnothing\left(\alpha_{\pi(q)}\left(\Gamma^{\lambda^{*}}(\mathscr{F})\right) \neq \varnothing\right)$ for all filters $\mathscr{F}$ on $S$.

Proof. If $\mathscr{F}$ is a filter on $S$ such that $\alpha_{q}\left(\Gamma^{\lambda^{*}}(\mathscr{F})\right)=\varnothing$, then let $T=S \cup\{a\}$, where $a$ is some element not in $S$. Define a convergence structure $p$ on $T$ as follows: $\mathscr{H} p$-converges to $a$ iff $\mathscr{H} \geqq \Gamma_{q}^{\sigma}(\mathscr{F}) \cap \dot{a}$ for $\sigma<\lambda^{*} ; \mathscr{H} p$-converges to $x, x \neq a$, iff $\mathscr{H} q$-converges to $x$. Using arguments similar to those of Theorem 2.4, we find that $p$ is $\lambda$-regular and $\Gamma(S)=T$, a contradiction. Hence $\alpha_{q}\left(\Gamma^{\lambda^{*}}(\mathscr{F})\right) \neq \varnothing$.

Conversely, let $(S, q)$ be embedded in $(T, p)$, where $(T, p)$ is $\lambda$-regular, and suppose that there is $x$ in $\Gamma(S)-S$. Then there exists an $\mathscr{F}$ on $S$ such that $\mathscr{F} p$-converges to $x$. Again, by analogy to the proof of Theorem 2.4 we have that $\alpha_{q}\left(\Gamma_{q}^{\lambda^{*}}(\mathscr{F})\right)=\varnothing$, a contradiction. Thus $(S, q)$ is $\lambda$-regular-closed.

The last two propositions of this section pertain to strongly regular spaces. Recall that a filter is closed if $\mathscr{F}=\hat{F}$ (or, equivalently, $\mathscr{F}=\Gamma(\mathscr{F})$ ). We will say that a maximal closed filter is ultraclosed. A simple application of Zorn's Lemma shows that every closed filter has an ultraclosed refinement. Hence we have the following proposition:

Proposition 2.11. The following statements about a strongly regular space $(S, q)$ are equivalent:

(i) $(S, q)$ is strongly-regular-closed.

(ii) For each filter $\mathscr{F}, \alpha_{q}(\hat{\mathscr{F}}) \neq \varnothing$.

(iii) Each ultraclosed filter converges.

Theorem 2.7 establishes that a $\lambda$-regular compact pseudo-topological space is minimal $\lambda$-regular, but for arbitrary convergence spaces the relationship between compactness and minimality for $\lambda$-regular spaces remains uncertain. The concluding proposition clarifies this relationship for strongly regular spaces.

A convergence space $(S, q)$ is said to be of type $\mathrm{Z}$ if a closed filter $\mathscr{F} q$-converges to $x$ whenever each ultrafilter finer than $\mathscr{F} q$-converges to $x$. Note that all pseudotopological spaces are of type $Z$.

Proposition 2.12. A compact strongly regular space is minimal strongly regular iff it is of type $\mathrm{Z}$.

Proof. Let $(S, q)$ be a compact strongly regular space of type Z. If $\{x\}=\alpha_{q}(\hat{\mathscr{F}})$ and $\mathscr{G}$ is an ultrafilter finer than $\hat{\mathscr{F}}$, then $\mathscr{G} q$-converges to $x$. Since $(S, q)$ is of type $Z$, $\hat{F} q$-converges to $x$, and hence the space is minimal strongly regular.

For the converse argument, let $q$ be minimal strongly regular and let $p$ be the convergence structure on $S$ defined as follows: $\mathscr{G} p$-converges to $x$ iff either $\mathscr{G}$ 
$q$-converges to $x$ or $\mathscr{G} \geqq \mathscr{F}$, where $\mathscr{F}$ is $q$-closed and each ultrafilter $\mathscr{H}$ finer than $\mathscr{F} q$-converges to $x$. One can verify by a direct argument that $p$ is the finest type $Z$ convergence structure coarser than $q$. Since $p$ and $q$ agree on ultrafilters, the closure operators relative to $p$ and $q$ coincide. Using this fact, it is easy to show that $p$ is strongly regular, and since $p \leqq q$, it follows that $p=q$ and $q$ is a convergence structure of type $\mathbf{Z}$.

Since the converse argument in the last proof did not require compactness, it follows that every minimal strongly regular space is of type $\mathbf{Z}$.

\section{Minimal Urysohn spaces.}

Definition 3.1. A convergence space $(S, q)$ is $\lambda$-Urysohn if, whenever $\mathscr{F} q$ converges to $x$ and $\mathscr{G} q$-converges to $y$, for $y \neq x$, it follows that $\Gamma^{\sigma}(\mathscr{F}) \vee \Gamma^{\sigma}(\mathscr{G})=\varnothing$ for each ordinal number $\sigma<\lambda^{*}$. An $\boldsymbol{\aleph}_{0}$-Urysohn space is said to be Urysohn; a space which is $\lambda$-Urysohn for all cardinal numbers $\lambda$ is said to be strongly Urysohn.

It is easy to see that a $\lambda$-regular space is $\lambda$-Urysohn (recall that we are considering only Hausdorff spaces); however the relationship between the corresponding minimal properties turns out to be just the reverse.

Proposition 3.2. A minimal $\lambda$-Urysohn space $(S, q)$ is minimal $\lambda$-regular.

Proof. Since $\lambda$-Urysohn spaces are more plentiful than $\lambda$-regular spaces, it is sufficient to show that $(S, q)$ is $\lambda$-regular. Let $\mathscr{F} q$-converge to $x$, and suppose that $\Gamma^{\beta}(\mathscr{F})$ does not $q$-converge for some $\beta<\lambda^{*}$. Define the convergence structure $p$ as follows: $\mathscr{H} p$-converges to $x$ iff $\mathscr{H} \geqq \mathscr{G} \cap \Gamma^{\beta}(\mathscr{F})$, where $\mathscr{G} q$-converges to $x ; \mathscr{H}$ $p$-converges to $y, y \neq x$, iff $\mathscr{H} q$-converges to $y$. One can show by a direct argument that $p$ is $\lambda$-Urysohn, and this contradiction establishes that $q$ is $\lambda$-regular.

Following Scarborough's approach [12], we call a filter $\mathscr{F}$ a $\lambda$-U-filter if $x \notin \alpha_{q}(\mathscr{F})$ and $\mathscr{G} q$-converges to $x$ imply that $\Gamma^{\sigma}(\mathscr{F}) \vee \Gamma^{\sigma}(\mathscr{G})=\varnothing$ for each $\sigma<\lambda^{*}$.

THEOREM 3.3. The following statements are equivalent:

(i) $(S, q)$ is minimal $\lambda$-Urysohn.

(ii) $\mathscr{F}$ q-converges to $x$ iff $\{x\}=\alpha_{q}\left(\Gamma^{\sigma}(\mathscr{F})\right)$ for each $\sigma<\lambda^{*}$.

(iii) If $(S, q)$ is $\lambda$-Urysohn and $\{x\}=\alpha_{q}(\mathscr{F})$, where $\mathscr{F}$ is a $\lambda$-U-filter, then $\mathscr{F} q$ converges to $x$.

Proof. (i) implies (ii). Let $\mathscr{F} q$-converge to $x$. If $y \neq x$ and $\mathscr{G} q$-converges to $y$, then $\Gamma^{\sigma}(\mathscr{G}) \vee \Gamma^{\sigma}(\mathscr{F})=\varnothing$ for each $\sigma<\lambda^{*}$. Hence $\{x\}=\alpha_{q}\left(\Gamma^{\sigma}(\mathscr{F})\right)$ for each $\sigma<\lambda^{*}$. If $\mathscr{F}$ fails to $q$-converge to $x$, then define $p$ as follows: $\mathscr{H} p$-converges to $x$ iff $\mathscr{H} \geqq \mathscr{F} \cap \mathscr{G}$, where $\mathscr{G} q$-converges to $x ; \mathscr{H} p$-converges to $y, y \neq x$, iff $\mathscr{H} q$-converges to $y$. It can be shown that $p$ is $\lambda$-Urysohn, a contradiction, and so $\mathscr{F}$ must $q$ converge to $x$.

(ii) implies (iii). Let $\mathscr{F} q$-converge to $x$ and $\rho<\lambda^{*}$. Then $\{x\}=\alpha_{q}\left(\Gamma^{\rho+\sigma}(\mathscr{F})\right)$ for all $\sigma<\lambda^{*}$. Hence $\Gamma^{o}(\mathscr{F}) q$-converges to $x$. Since (ii) implies the Hausdorff property, $(S, q)$ is $\lambda$-regular and thus $\lambda$-Urysohn. Consequently, if $\mathscr{F}$ is a $\lambda$-U-filter such that $\{x\}=\alpha_{q}(\mathscr{F})$, then $\{x\}=\alpha_{q}\left(\Gamma^{\sigma}(\mathscr{F})\right)$ for each $\sigma<\lambda^{*}$, and so by (ii) $\mathscr{F} q$-converges to $x$. 
(iii) implies (i). Let $p \leqq q$, where $p$ is $\lambda$-Urysohn, and let $\mathscr{F} p$-converge to $x$. Then $\{x\}=\alpha_{q}(\mathscr{F} \cap \dot{x})$. Let $\mathscr{G} q$-converge to a point $y$ distinct from $x$. Then $\mathscr{G} p$-converges to $y$ and thus $\Gamma_{p}^{\sigma}(\mathscr{G}) \vee\left(\Gamma_{p}^{\sigma}(\mathscr{F}) \cap \dot{x}\right)=\varnothing$. Consequently, $\Gamma_{q}^{\sigma}(\mathscr{G}) \vee\left(\Gamma_{q}^{\sigma}(\mathscr{F}) \cap \dot{x}\right)=\varnothing$ for each $\sigma<\lambda^{*}$, and we have that $\mathscr{F} \cap \dot{x}$ is a $\lambda$-U-filter. Thus $\mathscr{F} q$-converges to $x$ and $p=q$.

In particular, $(S, q)$ is minimal Urysohn iff it satisfies the condition: $\mathscr{F} q$ converges to $x$ iff $\{x\}=\alpha_{q}\left(\Gamma^{n}(\mathscr{F})\right)$ for all $n$ in $N$. A comparison of Theorems 2.4 and 3.3 shows a close kinship between minimal $\lambda$-regular and minimal $\lambda$-Urysohn spaces. By taking $\lambda$ sufficiently large, we see that a space is strongly $\lambda$-Urysohn iff it satisfies the condition: $\mathscr{F} q$-converges to $x$ iff $\{x\}=\alpha_{q}(\hat{\mathscr{F}})$. The next theorem is thus an immediate consequence of Corollary 2.6.

THEOREM 3.4. A convergence space is minimal strongly Urysohn iff it is minimal strongly regular.

THEOREM 3.5. A $\lambda$-Urysohn space is $\lambda$-Urysohn-closed iff, for each $\lambda$-U-filter $\mathscr{F}$, $\alpha_{q}(\mathscr{F}) \neq \varnothing$.

Proof. If $\alpha_{q}(\mathscr{F})=\varnothing$, then let $T=S \cup\{a\}, a \notin S$. Define $p$ on $T$ as follows: $\mathscr{H}$ $p$-converges to $a$ iff $\mathscr{H} \geqq \mathscr{F} \cap \dot{a} ; \mathscr{H} p$-converges to $x$ in $S$ iff $\mathscr{H}$ (regarded as a filter on $S$ ) $q$-converges to $x$. Since $\mathscr{F}$ is a $\lambda$-U-filter, we see that $p$ is $\lambda$-Urysohn and $\Gamma(S)=T$, a contradiction. Thus $\alpha_{q}(\mathscr{F}) \neq \varnothing$.

Let $(S, q)$ be embedded in $(T, p)$, where $(T, p)$ is $\lambda$-Urysohn, and let $x \in \Gamma(S)-S$. Then there is a filter $\mathscr{F}$ on $S$ such that $\mathscr{F} p$-converges to $x$. However $\mathscr{F}$ is a $\lambda$-Ufilter on $S$ relative to $q$, and $\alpha_{q}(\mathscr{F})=\varnothing$, a contradiction. Hence $(S, q)$ is $\lambda$-Urysohnclosed.

Proposition 3.6. Let $(S, q)$ be a minimal $\lambda$-Urysohn space. $A$ subset $A$ of $S$ is closed iff the subspace $\left(A, q_{A}\right)$ is minimal $\lambda$-Urysohn. For the sufficiency argument, one needs only that $(S, q)$ is $\lambda$-Urysohn.

Corollary 3.7. A minimal $\lambda$-Urysohn space is $\lambda$-Urysohn-closed.

Proposition 3.8. A compact pseudo-topological space is minimal $\lambda$-regular iff it is minimal $\lambda$-Urysohn.

From the study of minimal topological spaces it is known (see [12] and [13]) that there are noncompact minimal regular and minimal Urysohn topological spaces of all cardinalities greater than $\aleph_{0}$, and it is also known (see [13] and [14]) that countable minimal regular and minimal Urysohn topological spaces are necessarily compact. The corresponding results for convergence spaces are summarized in the next theorem and justified in the example which follows.

THEOREM 3.9. Let $\mu$ be an arbitrary cardinal number. Then there is a noncompact $\mathrm{T}_{2}$ convergence space $(S, p)$ of cardinality $\mu$ which is minimal regular, minimal Urysohn, and minimal strongly Urysohn. 
EXAMPLe 3.10. Let $(S, q)$ be the disjoint topological union of a countably infinite number of topological spaces $\left(S_{i}, q_{i}\right)$, where each $\left(S_{i}, q_{i}\right)$ is the one-point compactification of an infinite discrete space; for each $i$, let $x_{i}$ in $S_{i}$ be the compactification point. We divide the ultrafilters on $S$ into three classes:

Class 1. Those which contain exactly one of the $S_{i}$ 's.

Class 2. Those nonprincipal ultrafilters which contain a set $F$ which has a finite intersection with each $S_{i}$.

Class 3. Those such that each $F$ in $\mathscr{F}$ has an infinite intersection with infinitely many of the $S_{i}$ 's.

Let $p$ be the pseudo-topology on $S$ defined by specifying ultrafilter convergence as follows: For Class 1 ultrafilters, $p$-convergence agrees with $q$-convergence. For Class 2 ultrafilters, any Class 2 ultrafilter containing $X=\left\{x_{i}: i \in N\right\} p$-converges to $z_{0}$, where $z_{0}$ is some element of $S$ not in $X$, and each Class 2 ultrafilter not containing $X p$-converges to $y_{0}$, where $y_{0}$ is distinct from $z_{0}$ and also not in $X$. Each Class 3 ultrafilter fails to $p$-converge.

The cardinality of $S$ can be adjusted to any infinite cardinality $\mu$ by choosing each $S_{i}$ to have cardinality $\mu$.

Since Class 3 ultrafilters exist, $(S, p)$ is not compact. The $\mathrm{T}_{2}$ property can be verified by examining the neighborhoods of $y_{0}, z_{0}$, and the $x_{i}$ 's.

Note that the $p$-closure operator becomes idempotent in a finite number of steps. It follows from this fact and from several preceding theorems that minimal regular, minimal Urysohn, and minimal strongly regular are equivalent notions for $(S, p)$. Thus to show that $(S, p)$ has any one of these properties, it is sufficient to show that $\mathscr{F} p$-converges to $x$ iff $\{x\}=\alpha_{p}(\hat{F})$.

If $\mathscr{F}$ is a filter with a Class 3 ultrafilter refinement, then $\mathscr{F}$ cannot $p$-converge, and furthermore $y_{0}$ and $z_{0}$ are both in $\alpha_{p}(\hat{\mathscr{F}})$, so no problem arises. If $\mathscr{F}$ contains one of the $S_{i}$ 's, then it behaves like a filter on a compact Hausdorff topological space, so again no difficulty is encountered. The other possibility is a filter $\mathscr{F}$ which has no Class 3 ultrafilter refinement but which has the property that each $F$ in $\mathscr{F}$ meets more than one of the $S_{i}$ 's, and it can be shown by a direct argument that such a filter satisfies the specified condition.

\section{Minimal first countable spaces.}

Definition 4.1. A Hausdorff space $(S, q)$ is first countable if each filter which $q$-converges to $x$ contains a filter with a countable base (called a countable filter) which $q$-converges to $x$.

First countable convergence spaces have been investigated in [10] where they are shown to be closely related to Fréchet and sequential spaces; we show in this section that the same sort of relationship exists between minimal $T_{2}$ first countable spaces and minimal Fréchet spaces.

Most of the results of this section resemble those of $\S 1$, and proofs are omitted. 
THEOREM 4.2. A first countable space is minimal first countable iff it satisfies the following condition: A countable filter $\mathscr{F}$ q-converges to $x$ iff $\{x\}=\alpha_{q}(\mathscr{F})$.

THEOREM 4.2*. A $\mathrm{T}_{2}$ first countable space is minimal first countable $\mathrm{T}_{2}$ iff it satisfies the following condition: A countable filter $\mathscr{F} q$-converges to $x$ iff $\{x\}=\alpha_{\pi(q)}(\mathscr{F})$.

A convergence space is of type $\mathrm{W}$ if, whenever $\mathscr{F}$ is a countable filter and each ultrafilter finer than $\mathscr{F} q$-converges to $x$, then $\mathscr{F} q$-converges to $x$. Like the type $Z$ spaces of $\S 2$, type $\mathrm{W}$ spaces are generalizations of pseudo-topological spaces. Given a convergence space $(S, q)$, there is a finest type W convergence structure $p$ on $S$ which is coarser than $q$; if $q$ is first countable then so is $p$, and it follows that every minimal first countable space is of type $\mathrm{W}$.

Proposition 4.3. Each first countable compact space of type $\mathrm{W}$ is minimal first countable.

A space is countably compact [8, p. 162] if every sequence (or, equivalently, every countable filter) has an adherent point. It is an immediate consequence of Theorem 4.2 that every minimal first countable space is countably compact. One might hope that in Proposition 4.3 "compact" could be relaxed to "countably compact"; the following example, however, shows that this is not the case.

ExAmple 4.4. Let $S$ be the closed unit interval on the real line. We define a convergence structure $q$ on $S$ as follows: $\mathscr{F} q$-converges to $x$ iff $\mathscr{F}$ contains a countable filter $\mathscr{G}$ which contains a countable subset of $S$ and converges to $x$ relative to the usual topology on $S$. It is easy to verify that $(S, q)$ is a first countable countably compact space of type W. However, the usual neighborhood filter at any point $x$ in $S$ has $x$ as its only adherent point but (since it contains no countable set) fails to $q$-converge to $x$. Thus, by Theorem $4.2,(S, q)$ is not minimal first countable.

The proof of the next theorem is similar to that of Theorem 1.6.

THEOREM 4.5. A first countable $\left(\mathrm{T}_{2}\right)$ space $(S, q)$ is first-countable- $\left(\mathrm{T}_{2^{-}}\right)$closed iff $(S, q)((S, \pi(q)))$ is countably compact.

Thus each minimal first countable $\left(\mathrm{T}_{2}\right)$ space is first-countable- $\left(\mathrm{T}_{2^{-}}\right)$closed.

A pretopological space is Fréchet if, for each subset $A$, the closure of $A$ is precisely the set of limits of those countable filters which contain $A$; following our convention, we add the additional assumption that the space be Hausdorff. Theorem 2 of [10] (where the spaces involved are not assumed to be Hausdorff) states that a pretopological space is Fréchet iff $p$ is the topological modification of a first countable convergence structure $q$ on $S$. A simple Hausdorffization of this result can be stated as follows.

Proposition 4.6. A pretopological space is Fréchet iff it is the pretopological modification of a first countable $\mathrm{T}_{2}$ convergence space. 
In the "necessity" part of the proof of this proposition, one starts with a Fréchet space $(S, p)$ and constructs a first countable convergence structure $q$ on $S$ as follows: A filter $\mathscr{F} q$-converges to $x$ iff it contains some countable filter which $p$-converges to $x$. By this construction, $q$ is the coarsest first countable convergence structure finer than $p$, and since $p$ is uniquely determined by the convergence of countable filters, it follows that for any other Fréchet space $p_{1}<p$, we have $q_{1}<q$, and vice versa.

THEOREM 4.7. The following statements about a Fréchet space are equivalent:

(i) $(S, p)$ is minimal Fréchet.

(ii) $(S, p)$ is the pretopological modification of a minimal first countable $\mathrm{T}_{2}$ space.

(iii) A countable filter $\mathscr{F} p$-converges to $x$ iff $\{x\}=\alpha_{p}(\mathscr{F})$.

Proof. From the remarks which precede the theorem, $p$ is minimal Fréchet iff $q$ is minimal first countable $\mathrm{T}_{2}$, and so (i) and (ii) are equivalent. By Theorem 4.2*, the statement that $q$ is minimal first countable $\mathrm{T}_{2}$ is equivalent to saying: for countable filters $\mathscr{F}, \mathscr{F} q$-converges to $x$ iff $\{x\}=\alpha_{\pi(q)}(\mathscr{F})$. But $\pi(q)=p$, and $p$ convergence coincides with $q$-convergence on countable filters; thus the specified condition is equivalent to (iii).

Concluding Remarks. There are a number of questions which remain to be answered. We formulate three such questions in terms of an arbitrary property $P$.

(1) Is a product of minimal $P$ spaces again a minimal $P$ space?

(2) Is there a minimal $\mathrm{P}$ convergence structure on a set $S$ coarser than some given convergence structure with property $\mathbf{P}$ ?

(3) Can a given convergence space with property $\mathbf{P}$ be densely embedded in a minimal $\mathbf{P}$ convergence space?

If $\mathrm{P}$ is the Hausdorff property, the answer to the first two questions is affirmative. Also question three has an affirmative answer for the Hausdorff case iff the given convergence space is pseudo-topological. This can be deduced from the results found in [11]. For $T_{2}$ spaces, the answer is affirmative for (1) and negative for (2). The answer to (1) is affirmative for first countable spaces if the number of spaces involved in the product is countable. The remaining cases belong to the category of unsolved problems.

Acknowledgement. The authors wish to thank the referee for several helpful suggestions.

\section{REFERENCES}

1. M. P. Berri, Minimal topological spaces, Trans. Amer. Math. Soc. 108 (1963), 97-105. MR 27 \#711.

2. M. P. Berri and R. H. Sorgenfrey, Minimal regular spaces, Proc. Amer. Math. Soc. 14 (1963), 454-458. MR 27 \#2949.

3. H. J. Biesterfeldt, Jr., Regular convergence spaces, Nederl. Akad. Wentensch. Proc. Ser. A $69=$ Indag. Math. 28 (1966), 605-607. MR 34 \#5048.

4. C. H. Cook and H. R. Fischer, Regular convergence spaces, Math. Ann. 174 (1967), 1-7. MR 37 \#5837. 
5. H. R. Fischer, Limesräume, Math. Ann. 137 (1959), 269-303. MR 22 \#225.

6. B. V. Hearsey, On extending regularity and other topological properties to convergence spaces, Ph.D. Dissertation, Washington State University, Pullman, Washington, 1968.

7. H. Herrlich, $T_{v}$-Abgeschlossenheit und $T_{v}$-Minimalität, Math. Z. 88 (1965), 285-294. MR $32 \# 1664$.

8. J. L. Kelley, General topology, Van Nostrand, Princeton, N. J., 1955. MR 16, 1136.

9. D. C. Kent, Convergence quotient maps, Fund. Math. 65 (1969), 197-205. MR 40 \#3497.

10. - Decisive convergence spaces, Fréchet spaces and sequential spaces, Rocky Mt. J. Math. 1 (1971), 367-374.

11. G. D. Richardson, A Stone-Čech compactification for limit spaces, Proc. Amer. Math. Soc. 25 (1970), 403-404.

12. C. T. Scarborough, Minimal Urysohn spaces, Pacific J. Math. 27 (1968), 611-617. MR 38 \#6530.

13. C. T. Scarborough and A. H. Stone, Products of nearly compact spaces, Trans. Amer. Math. Soc. 124 (1966), 131-147. MR 34 \#3528.

14. R. M. Stephenson, Jr., A countable minimal Urysohn space is compact, Proc. Amer. Math. Soc. 22 (1969), 625-626. MR 39 \#6255.

Washington State University, Pullman, Washington 99163

East Carolina University, Greenville, North Carolina 27834 